\title{
O ENFRENTAMENTO DA EQUIPE DE ENFERMAGEM EM ATENDIMENTOS A PACIENTES EM CRISE PSICÓTICA
}

\author{
NURSING TEAM COPING IN CARE OF PSYCHOTIC PATIENTS
}

\author{
Samara de Oliveira ${ }^{\mathrm{a}^{*}}$, Wellington Fernando da Silva Ferreira ${ }^{\mathrm{b}^{* *}}$, Cláudia Ribeiro de \\ Vasconcelos $^{\mathrm{c}^{*}}$, Denecir de Almeida Dutra ${ }^{\mathrm{d}^{*}}$ \\ asamarinhaeira@gmail.com, bwellingtonferreira42@gmail.com, cprofe.cv@hotmail.com, ddenecir.dutra@terra.com.br \\ ${ }^{*}$ Centro Universitário Campos de Andrade - Curitiba (PR), Brasil \\ **Faculdade Unyleya - Brasília (DF), Brasil
}

Data de recebimento de artigo: 11/03/2017

Data de aceite do artigo: 26/07/2017

\section{RESUMO}

Introduçáo: Estudo centrado no atendimento às emergências psiquiátricas em hospitais gerais. Objetivo: Caracterizar o enfrentamento de uma equipe de enfermagem perante atendimentos de emergência a pacientes em crise psicótica em um hospital do município de Contenda (PR). Métodos: Realizou-se pesquisa de campo quantiqualitativa, descritiva e transversal com 20 participantes da equipe de enfermagem que atuam diretamente com pacientes em crise psicótica na emergência da instituição. Resultados: Predominaram auxiliares de enfermagem do gênero feminino, com média de idade de 40 anos, tempo de atuação de 12 anos e média de 8 anos na instituição. Destacam-se a falta de habilidade e insegurança diante de uma emergência de crise psicótica, sendo atendimento individual ou em grupo. Os participantes ressaltam a importância de educação continuada, relacionada à carência de literatura sobre o tema, de cursos e de treinamentos. Conclusão: Evidenciou-se a necessidade de educaçáo continuada para criar condiçôes de atendimento em emergências psiquiátricas e despertar o interesse nos profissionais sobre a temática, conscientizando-os e incentivando atendimento humanizado ao paciente com transtorno mental.

Palavras-chave: Crise psicótica; emergência psiquiátrica; enfermagem.

\begin{abstract}
Introduction: A study focused on psychiatric emergency care in general hospitals. Objective: Aiming at characterizing coping mechanisms of a nursing team regarding emergency care of patients in psychotic episodes in a hospital in the municipality of Contenda (Paraná, Brazil). Methods: A quantitative, qualitative, descriptive, and cross-sectional field study was performed with 20 participants of the nursing team who work directly with patients in psychotic episode in the institution's emergency room. Results: Auxiliary nurses of the female gender predominated, with a mean age of 40 years old, with a 12-year-old working time, and an average of 8 years in the institution. Lack of skill and insecurity in the context of psychotic episode emergency stand out, in individual or group care. The participants emphasize the importance of continuing education, related to the lack of literature on the subject and absence of courses or training. Conclusion: The necessity of continuous education has become evident to create conditions for psychiatric emergency care and stimulate the interest of professionals in this subject, making them aware and encouraging a humanized care to the patient with mental disorder.
\end{abstract}

Keywords: Psychotic episode; psychiatric emergency; nursing. 


\section{Introdução}

O perfil epidemiológico das emergências psiquiátricas (EP) vem mudando de forma significativa nos últimos anos em virtude da reforma psiquiátrica, alterando a rotina das equipes e dos serviços de saúde. Dessa forma, atualmente, as situaçôes de crise psicótica são inicialmente atendidas em serviços de emergência, com destaque aos hospitais gerais. Os objetivos dos primeiros cuidados são estabilização do quadro, estabelecimento de hipótese diagnóstica, exclusão de causa orgânica e encaminhamento para continuidade no tratamento ${ }^{1,2}$.

Os hospitais gerais, conforme Portaria $n^{\circ}$ 3.088/2011 do Ministério da Saúde (MS) ${ }^{3}$, são pontos de atenção de urgência/emergência em saúde mental, compondo a Rede de Atenção Psicossocial (RAPS) em substituição ao modelo manicomial, portanto relevantes ao cuidado de pessoas em sofrimento psíquico intenso. De acordo com o Decreto no 7.508/2011, as urgências/emergências são portas de entrada do Sistema Único de Saúde (SUS), assim como a atenção primária e psicossocial, que se completam na rede ampliada de saúde, possibilitando a resolução das demandas da populaçãó .

A crise psicótica é definida como uma dissociação da estrutura psíquica, fazendo com que o indivíduo desencadeie discursos e comportamentos desorganizados devido à alteração do pensamento (delírios) e/ou da sensopercepção (alucinaçóes), comuns na esquizofrenia, no transtorno afetivo bipolar, na depressão psicótica e nas demências, e devido ao abuso de substâncias psicoativas (SPA). É durante um surto psicótico que ocorre perda da realidade, podendo colocar a própria pessoa e outras à sua volta em risco ${ }^{5,6}$.

Segundo a American Psychiatric Association (APA) ${ }^{7}$, delírios são falsas crenças baseadas na inferência incorreta da realidade, e alucinaçóes são percepçóes de estímulos inexistentes, podendo ocorrer em qualquer modalidade sensorial (auditiva, visual, tátil, palativa, olfativa). São sintomas psicóticos precipitantes de agitação psicomotora, autoagressividade e heteroagressividade.

A esquizofrenia é responsável por proporção importante de visitas às emergências. Trata-se de um transtorno mental grave e persistente, caracterizado primordialmente pela presença de sintomas psicóticos que causam impacto cognitivo, afetivo e funcional. Embora existam vários subtipos de esquizofrenia e especificidades, em geral, no curso da doença são recorrentes períodos de crise que demandam intervençóes terapêuticas imediatas ${ }^{8,9,10}$.

$\mathrm{Na}$ depressão psicótica, segundo a Organização Mundial da Saúde (OMS) ${ }^{11}$, podem ocorrer alucinações, delírios e lentidão psicomotora de gravidade tal que as atividades sociais se tornam prejudicadas, além de risco de suicídio, desidratação e desnutrição.

Quanto ao transtorno afetivo bipolar, a OMS classifica os episódios psicóticos tanto na fase maníaca quanto na fase depressiva. Na mania pode haver a presença de ideias delirantes (em geral de grandeza), alucinaçóes (em geral auditivas) e fuga de ideias de gravidade tal que a pessoa se torna incompreensível ou inacessível à comunicação coerente. $\mathrm{Na}$ fase depressiva, os sintomas são semelhantes aos da depressão psicótica ${ }^{11}$.

Os quadros de demência são parte de um conjunto de doenças crônicas e progressivas que envolvem complicaçôes tanto clínicas quanto psiquiátricas, tais como declínio cognitivo, sintomas psicóticos e alteraçóes comportamentais, comprometendo as atividades de vida diária e evoluindo para perda da autonomia e total dependência, o que leva o seu portador a necessitar de atendimento hospitalar em vários momentos, inclusive em situaçôes de crise psicótica ${ }^{12,13}$.

No que diz respeito às SPA, os sintomas psicóticos ocorrem durante ou imediatamente após o consumo de drogas. Esse estado se caracteriza pela presença de alucinações (tipicamente auditivas, mas frequentemente polissensoriais), delírios (regularmente do tipo persecutório) e afetos anormais, podendo ir de um medo intenso ao êxtase. Além disso, destacam-se quadros psicóticos na abstinência de algumas $\mathrm{SPA}^{7,8,11}$.

As EP são um problema de saúde pública, sendo, portanto, tema relevante para profissionais da saúde de modo geral e para as equipes de enfermagem emergencistas, em especial, por terem contato próximo a pacientes em momentos críticos. O estigma, as concepçóes errôneas e a falta de treinamento são fontes de insegurança na avaliação e manejo de quadros que exijam açôes rápidas, eficientes e eficazes, tornando-se relevante que os trabalhadores estejam capacitados para um atendimento integral e humanizado ${ }^{10}$.

A assistência a vulneráveis é tema que precisa ser discutido no meio acadêmico, de forma a contribuir para o desenvolvimento do pensamento crítico e científico em contraponto ao senso comum. É imprescindível que a enfermagem esteja preparada, tanto técnica como emocionalmente, para promover cuidados aos portadores de transtornos mentais e seus familiares, compreendendo seu espaço de atuação e construindo uma práxis mais eficaz no que se refere aos atendimentos de $\mathrm{EP}^{10}$.

Diante do exposto, considera-se essencial conhecer como a enfermagem percebe a própria conduta, o preparo e a disponibilidade emocional em relação a crises psicóticas admitidas na emergência, evidenciando a atual organizaçáo da assistência no contexto da saúde mental no Brasil. 
O objetivo deste estudo foi caracterizar o enfrentamento de uma equipe de enfermagem perante atendimentos de emergências a pacientes em crise psicótica em um hospital do município de Contenda (PR).

\section{Materiais e métodos}

Trata-se de estudo quantiqualitativo, descritivo e transversal realizado em um renomado hospital do município de Contenda (PR) especializado em atendimentos a situações de urgência/emergência. A amostra foi composta por 20 trabalhadores da enfermagem que atuam diretamente com pacientes em crise psicótica na emergência, conforme autorização da instituição coparticipante. A coleta de dados foi realizada entre os meses de agosto e setembro de 2016. A declaração para solicitação de coparticipação na pesquisa está documentada.

$\mathrm{O}$ projeto foi submetido à avaliação do Comitê de Ética em Pesquisa com Seres Humanos do Centro Universitário Campos de Andrade e autorizado conforme parecer consubstanciado ${ }^{\circ} 1.700 .353$. Após autorização, foi agendada reuniáo com a gerente de enfermagem do hospital para solicitar a indicação aleatória de participantes que atendessem aos critérios do estudo.

Como critérios de inclusão, foram estabelecidos: atuar na emergência do local escolhido para a pesquisa como enfermeiro, auxiliar ou técnico de enfermagem; ser funcionário desse hospital há pelo menos seis meses; estar em condição de ser entrevistado; concordar em participar do estudo em todas as etapas; e assinar o Termo de Consentimento Livre e Esclarecido, respeitando o estabelecido nas normatizaçôes éticas referentes à Resolução no 466/2012 do Conselho Nacional de Saúde ${ }^{14}$. Já de acordo com os critérios de exclusão, foram preteridos os trabalhadores que solicitaram transferência a outro setor, que estavam de férias ou afastados do ambiente de trabalho.

A coleta de dados foi realizada por meio de um questionário semiestruturado, com perguntas abertas e fechadas, nas dependências da instituição coparticipante em um único encontro de aproximadamente 15 minutos com cada entrevistado. $\mathrm{O}$ anonimato foi assegurado pela identificação dos auxiliares de enfermagem com a letra "A", dos técnicos de enfermagem com a letra "T" e dos enfermeiros com a letra "E". O questionário foi realizado em três etapas: na primeira, dados demográficos do participante foram obtidos por meio de (cinco) perguntas objetivas sobre gênero, idade, atuação profissional (se enfermeiro, auxiliar ou técnico de enfermagem), tempo de atuação na instituição e tempo de trabalho na área. $\mathrm{Na}$ segunda, foram apresentadas (seis) perguntas objetivas em forma de quadro, contendo (três) alternativas cada para escolha daquela que melhor se encaixasse conforme cada indivíduo (sim, não e talvez). As perguntas englobam aspectos relacionados ao preparo do trabalhador para atender pacientes e familiares em crise psicótica na emergência, se a admissão desses pacientes interfere no emocional da equipe e se já tiveram treinamento, orientação ou informação sobre a temática.

Por fim, na terceira, foram feitas (quatro) perguntas subjetivas sobre conhecimento, enfrentamento e necessidade do preparo da equipe de trabalho diante da crise psicótica.

Os dados objetivos são apresentados através de estatística básica, com números absolutos $(\mathrm{N})$ e relativos (\%), enquanto os dados subjetivos foram transcritos por meio da teoria de Bardin ${ }^{15}$, realizando pré-análise, exploração do material, tratamento dos dados obtidos e interpretação aprimorada, categorizando as temáticas e transcrevendo os relatos conforme registrados pelos participantes.

\section{Resultados}

As variáveis do perfil geral dos trabalhadores participantes da pesquisa e as do enfrentamento da equipe de enfermagem em atendimentos a crises psicóticas são registradas em formato de tabela para facilitar a visualização e a análise dos resultados. Os números relativos (\%) foram calculados a partir dos números absolutos $(\mathrm{N})$.

Tabela 1: Variáveis do perfil geral dos trabalhadores da enfermagem de Contenda (PR), 2016.

\begin{tabular}{lcc} 
Variáveis & N & \% \\
Gênero & & \\
Feminino & $\mathbf{1 7}$ & $\mathbf{8 5}$ \\
Masculino & 3 & 15 \\
Faixa etária & & \\
20-30 & 2 & 10 \\
$31-41$ & $\mathbf{9}$ & $\mathbf{4 5}$ \\
42-52 & 7 & 35 \\
53-60 & 2 & 10 \\
Atuaçáo profissional & & \\
Auxiliar de enfermagem & $\mathbf{9}$ & $\mathbf{4 5}$ \\
Técnico de enfermagem & 7 & 35 \\
Enfermeiro & 4 & 20 \\
Tempo em que atua na enfermagem (anos) & & \\
2-11 & $\mathbf{1 2}$ & $\mathbf{6 0}$ \\
12-21 & 6 & 30 \\
22-30 & 2 & 10 \\
Tempo em que atua na instituiçáo (anos) & & \\
1-10 & $\mathbf{1 5}$ & $\mathbf{7 5}$ \\
11-20 & 4 & 20 \\
21-30 & 1 & 5 \\
TOTAL & 20 & 100 \\
\hline & & \\
\hline
\end{tabular}


Conforme a Tabela 1, observa-se que as idades dos participantes variam entre 20 e 60 anos, com uma média de 40 anos, e há predomínio do gênero feminino e de auxiliares de enfermagem.
O tempo de atuação em enfermagem varia de 2 a 30 anos, com média de 12 anos. Quanto ao tempo de atuação na instituição, há uma duração de 1 a 30 anos, com média 8 de anos.

Tabela 2: Variáveis do preparo da equipe de enfermagem de Contenda (PR) diante de crise psicótica, 2016.

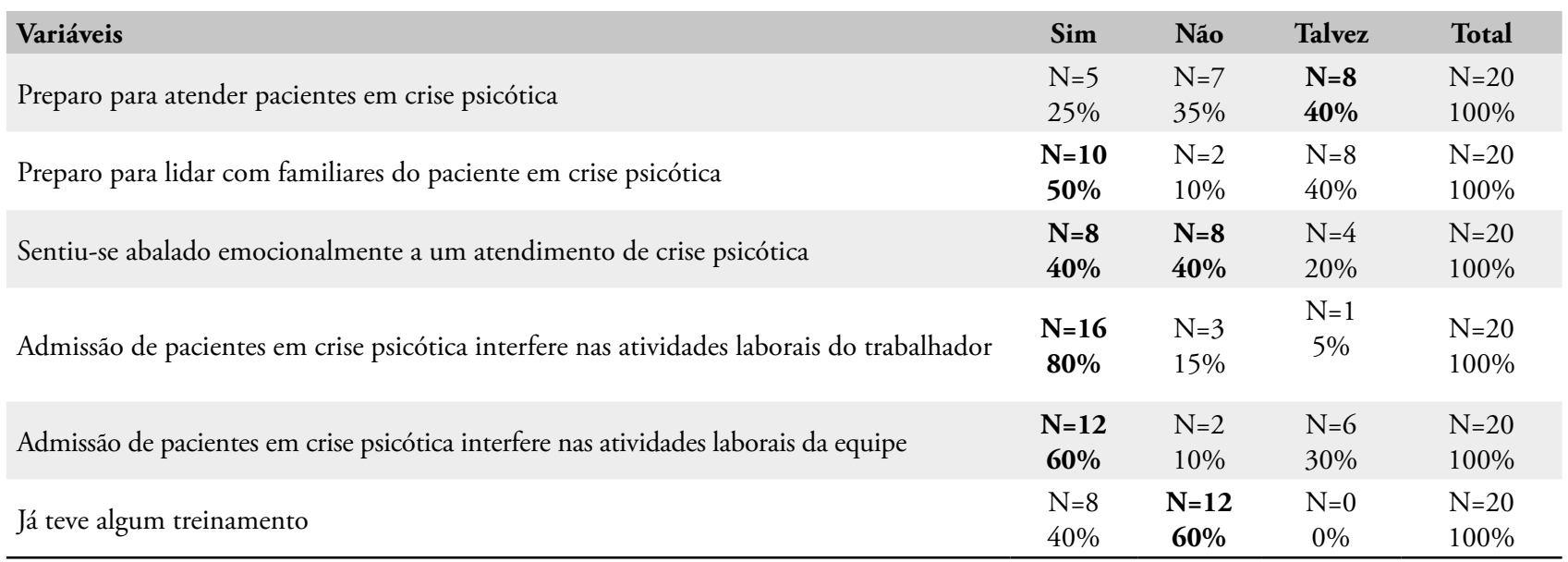

Conforme a Tabela 2, a maioria dos entrevistados se autoavalia como despreparado ou em dúvida sobre o seu preparo diante de uma crise psicótica, mas acredita que consegue lidar com familiares desses pacientes com mais facilidade, embora a dúvida permaneça em uma parcela. Em relação ao emocional, uma porcentagem demonstrou-se abalada diante desses atendimentos, declarando que interferem nas suas atividades e dos seus colegas de forma significativa. A maioria assinala que não teve nenhum treinamento específico para atendimentos de crise psicótica.

\section{Aspectos subjetivos sobre o enfrentamento diante da crise psicótica}

Considerando as variáveis quanto à definição da crise psicótica, a maioria dos participantes conseguiu descrever conceitos básicos relacionados a fatores de risco, tais como transtornos mentais e intoxicação por substâncias psicoativas, além de identificar características relacionadas ao exame do estado mental, conforme a fala a seguir:

A psicose pode ser derivada de caráter psiquiátrico, como psicopatologia, como por uso de entorpecentes e afins. Que pode se manifestar nos pacientes de diversas formas, agitação, confusão, agressividade, fuga, isolamento. (E3)

Três participantes demonstraram desconhecimento sobre a crise psicótica e seus sintomas:
Perda de seu caráter, vive mundo irreal, diferente de doenças emocionais. (A4)

Muito pouco. (A8)

Pouca coisa. (T1)

Porém, quanto ao enfrentamento dos participantes da pesquisa diante da crise psicótica, identificaram-se as categorias medo, despreparo e qualificação, com predomínio da última.

$\mathrm{Na}$ categoria medo, destacou-se:

Fico com medo e sem atitudes. (A8)

Fico com medo porque a maioria é agressiva e fica fora de si. (T4)

Os trabalhadores que mencionaram despreparo parecem valorizar o treinamento como uma solução para melhor enfrentamento desses atendimentos.

Nunca tivemos treinamentos de crises psicóticas, então não gosto desses atendimentos. (A5)

Não temos um preparo adequado para este enfrentamento, precisamos de treinamentos para melhorar os nossos conhecimentos e elaborar fórmulas e maneiras de termos este enfrentamento mais eficiente. (T3)

$\mathrm{Na}$ categoria qualificação, apareceram palavras como controle emocional, respeito, ética e agilidade.

Atendo com atenção, respeito e ética, tentado auxiliar na melhor forma possivel. (A1) 
Controle no momento do cuidado desse paciente, respeito, agilidade em serviço com medicamentos e percepção na admissão deste paciente. (T6)

Quanto ao enfrentamento de sua equipe, a maior parte dos participantes refere despreparo, falta de conhecimento, insegurança e medo. Porém, uma pequena parcela (20\%) afirma habilidade e entrosamento.

Relatos de medo da equipe:

Ficamos com receio, pois alguns são agressivos. (A5) Ficam com o pé atrás, pois acredito que tenham o medo que eu tenho. (T4)

Falas que simbolizam despreparo:

Alguns não sabem lidar e acabam não exercendo com ética seu atendimento. (A1)

Muitos não respeitam, sentem ofendidos e acham que o paciente está se fazendo para chamar a atenção. (A4)

Bastante despreparo. (T2)

Falta preparo geral. (E2)

Alguns relatam entrosamento e habilidade:

Com habilidade, a equipe já tem um excelente entrosamento e conhecimento inclusivo no manejo a este paciente. (E3) Com entrosamento da nossa equipe mantemos a agilidade para não piorar o quadro e ajudar o máximo possivel. (A6)

Foi de opinião unânime a necessidade de uma intervenção no local de trabalho a respeito dos atendimentos a crises psicóticas, pois acreditam que o preparo da equipe pode melhorar com cursos, treinamentos, palestras e a implantação de educação continuada no local da pesquisa.

Sim, com treinamentos e melhor entrosamento de equipe e chefia. (A2)

Sim, com educação continuada podemos rever e atualizar nossos conhecimentos. (A6)

Sim, fazer cursos para aperfeiçoamento das equipes. (A8)

Sim, se tivéssemos maior abordagem desse assunto, acredito que nosso pensamento mudasse em relação aos doentes. (T4) Sempre, continuamente. Nesta área de atuação nunca estamos completamente atualizados e nunca nos deparamos com todas as situaçóes possiveis. Reciclagens com profissional capacitado e com maior experiência (grande PS ou hospitais psiquiátricos) seriam bem-vindas. (E3)

\section{Discussão}

De acordo com os resultados deste estudo, verificou-se despreparo de parcela significativa de trabalhadores em lidar com os pacientes em crise psicótica, o que pode estar relacionado à formação profissional ineficaz, seja pelo ensino formal técnico e superior ou pela carência de literatura sobre o tema e de cursos ou treinamentos, confirmada por outros estudos semelhantes ${ }^{16}$.

De acordo com a pesquisa de Kondo et al. ${ }^{17}$, realizada em uma unidade de pronto-atendimento na cidade de Curitiba (PR), toda a equipe de enfermagem participante relatou dificuldades no atendimento de EP. Caveião et al. ${ }^{18}$ reforçam que muitos profissionais de enfermagem emergencistas não se sentem capacitados para o manejo de pacientes portadores de transtornos mentais.

Metade da equipe demonstra despreparo para abordar e acolher os familiares desses pacientes que estáo passando por momentos de sofrimento e desestruturação. De acordo com Tavares et al. ${ }^{19}$, a enfermagem deve ampliar seus conhecimentos sobre a família, discutindo ensinamentos sobre as situaçóes impostas pelo transtorno mental e intervindo no processo de cuidar, visto que a abordagem correta do familiar é fundamental para o quadro clínico desse paciente.

Para Silva et al. ${ }^{20}$, a equipe de enfermagem tem a necessidade de controlar suas emoçóes e sentimentos diante de um paciente psiquiátrico para haver um bom desempenho em uma situaçáo emergencial. Segundo os autores, esse controle pode estar relacionado à insegurança, ao medo e ao pensamento de incapacidade, reafirmando os achados de grande parte da amostra ao avaliar a interferência no seu próprio desempenho profissional e no de seus colegas nos atendimentos a crises.

A maioria dos participantes soube descrever conceitos e manifestaçóes de crises psicóticas, sinalizando um conhecimento básico sobre psicopatologia, o que influencia positivamente no cuidado recebido pelo paciente ${ }^{16}$.

Porém, embora alguns participantes tenham relatado habilidade e entrosamento da equipe nos atendimentos à crise, a maioria revelou dificuldade. Assim, compreende-se que essa amostra identifica e define a crise psicótica, porém apresenta uma assistência falha permeada por medo, despreparo e falta de ética. Segundo a pesquisa de Kondo et al. ${ }^{17}$, os sentimentos negativos relatados pelos profissionais de enfermagem em relação ao paciente com transtorno mental são resultado da história da saúde mental, em uma sociedade em que os loucos devem permanecer distantes de todos. Conclui-se, então, que esses sentimentos só afastam a equipe do paciente em crise psicótica, negligenciando os cuidados prestados.

Identificou-se como opinião unânime entre os entrevistados que uma intervenção seria importante para os atendimentos à crise psicótica, sendo fundamental educação continuada no local, como palestras, cursos e treinamentos. Para Lima et al. ${ }^{21}$, a capacitação ocorre através da combinação de recursos e do trabalho conjunto entre os profissionais, mantendo uma estrutura 
adequada para as crises psicóticas. Santos et al. ${ }^{22}$ reforçam que, para capacitar seus funcionários na área de EP, os gestores deveriam implantar um treinamento em saúde mental que se iniciasse nas políticas que modificaram essa área, passando para o cuidado no atendimento e alcançando, assim, o objetivo de qualificação.

Para Borges et al. ${ }^{23}$, Ferreira et al. ${ }^{24}$ e Barbosa et al. ${ }^{25}$, o ensino superior amplia o entendimento da enfermagem trazendo benefícios e progressos, expondo ao profissional a maneira que irá vivenciar o atendimento, modificando conceitos sobre a temática, proporcionando a quebra desse preconceito, conduzindo o profissional ao conhecimento satisfatório.

\section{Conclusão}

Diante dos transtornos mentais, a saúde e seu subcampo avançaram muito nas últimas décadas, porém a enfermagem e a saúde mental são uma intersecção dos cuidados do processo saúde-doença ligados aos transtornos mentais.

Nesse sentido, as abordagens que estão intercaladas entre psicologia, medicina e enfermagem possuem intrinsecamente peculiaridades, porém, na enfermagem alia-se o contato direto do paciente e, consequentemente, respalda-se na Sistematização da Assistência de Enfermagem vigente e ligada à crise psicótica.

Assim, considerando os resultados da pesquisa, nota-se o despreparo diante de um atendimento emergencial à crise, o que é relacionado à formação profissional ineficaz e à falha na educação continuada, além da carência de literatura sobre o tema e ausência efetiva de cursos recicladores dos trabalhadores do setor.

Para remodelar essa realidade, é necessário que os gestores tenham educação continuada com espaço para a crise psicótica, construindo condiçôes para atendimentos em EP e tendo em mente que somente a técnica e as metodologias vigentes não dáo conta de ensinar conceitos básicos, manejo e percepçóes de respeito.

$\mathrm{O}$ processo de aprendizagem da ciência deve auxiliar nessa transformação, pois é em sala de aula que são discutidos sentimentos que aparecem nos atendimentos, de modo a modificar a percepçáo e melhorar as opinióes errôneas que envolvem esses pacientes, aproximando teoria e prática.

Nesse contexto, também surge a necessidade de despertar interesse nos profissionais envolvidos e nas instituições de saúde de atendimentos de emergência sobre a temática, conscientizando e incentivando um atendimento humanizado ao paciente com transtorno mental.

Este estudo possibilitou compreender os sentimentos que a equipe de enfermagem tem diante da crise psiquiátrica, demonstrando que ampliar seu conhecimento sobre a crise mudaria aspectos mínimos e necessários para uma transformação da qualidade de vida do paciente.

\section{Referências}

1. Quevedo J, Carvalho AF. Emergências Psiquiátricas. $3^{a}$ ed. Porto Alegre: Artmed; 2014. 336p.

2. Santa Catarina. Urgências e emergências psiquiátricas em pronto-socorro: protocolo de acolhimento e regulação. Florianópolis: Governo do Estado de Santa Catarina; 2015.

3. Brasil. Ministério da Saúde. Portaria no 3.088, de 23 de dezembro de 2011. Institui a Rede de Atenção Psicossocial para pessoas com sofrimento ou transtorno mental e com necessidades decorrentes do uso de crack, álcool e outras drogas, no âmbito do Sistema Único de Saúde (SUS) [Internet]. Diário Oficial da União. Brasília, DF; 26 dez 2011 [citado em 2016 mar 17]. Seção 1, p 230. Disponível em: https://goo.gl/bi9LqJ

4. Brasil. Decreto no 7.508, de 28 de junho de 2011. Regulamenta a Lei no 8.080, de 19 de setembro de 1990, para dispor sobre a organizaçáo do Sistema Único de Saúde - SUS, o planejamento da saúde, a assistência à saúde e a articulação interfederativa, e dá outras providências [Internet]. Diário Oficial da União. Brasília, DF; 29 jun 2011 [citado em 2016 mar 17]. Seção 1, p. 1. Disponível em: https://goo.gl/x1U24Q.

5. Vieira A, Moreira JI, Loureiro K, Morgadinho R. Esquizofrenia e outras perturbaçóes psicóticas. O Portal dos Psicólogos [Internet]. 2010 [citado em 2017 set 26]. Disponível em: https://goo.gl/FrEkvV

6. Sucar DD, Sougey EB, Neto JB. Surto psicótico pela possível interaçáo medicamentosa de sibutramina com finasterida. Rev Bras Psiquiatr. 2002;24(1):30-3.

7. American Psychiatric Association. Manual diagnóstico e estatístico de transtornos mentais: DSM-5. $5^{\mathrm{a}}$ ed. Porto Alegre: Artmed; 2014. 948p.

8. Toy EC, Klamen D. Casos clínicos em psiquiatria. 4a ed. Porto Alegre: AMGH; 2014. 496p.

9. Sanches M, Uchida RR, Tamai S. Manejo do paciente psiquiátrico grave. São Paulo: Roca, 2009. 176p.

10. Carvalho MB, Santos AML. Emergências Psiquiátricas. In: Carvalho $\mathrm{MB}$, organizadora. Psiquiatria para a enfermagem. São Paulo: Rideel; 2012. p. 279-92.

11. Organização Mundial da Saúde. CID-10: classificação estatística internacional de doenças e problemas relacionados à saúde [Internet]. $10^{\mathrm{a}}$ rev. Versão 2008 [citado em 2017 set 26]. v. 1. Disponível em: https://goo.gl/JWRHFh 
12. Schlindwein-Zanini R. Demência no idoso: aspectos neuropsicológicos. Rev Neurocienc. 2010;18(2):220-6.

13. Pereira LSM, Soares SM. Fatores que influenciam a qualidade de vida do cuidador familiar do idoso com demência. Ciênc Saúde Coletiva. 2015;20(12):3839-51.

14. Brasil. Conselho Nacional de Saúde. Resolução no 466, de 12 de dezembro de 2012. Aprova diretrizes e normas regulamentadoras de pesquisas envolvendo seres humanos. Diário Oficial da União. Brasília, DF; n. 12, 13 jun 2013. Seção 1, p. 59.

15. Bardin L. Análise de conteúdo. São Paulo: Ediçôes 70; 2011. 280p.

16. Ikuta CY, Santos MA, Badagnan HF, Donato ECSG, Zanetti ACG. Conhecimento dos profissionais de enfermagem em situaçóes de emergência psiquiátrica: revisão integrativa. Rev Eletr Enf. 2013;15(4):1034-42.

17. Kondo EH, Vilella JC, Borba LO, Paes MR, Maftum MA. Abordagem da equipe de enfermagem ao usuário na emergência em saúde mental em um pronto atendimento. Rev Esc Enferm USP. 2011;45(2):501-7.

18. Caveiáo C, Hey AP, Montezeli JH, Sales WB, Visentin A, Kaled M. Portador de transtorno mental em situação de emergência: dificuldades de atendimento percebidas pela equipe de enfermagem em uma unidade mista. Cad Esc Saúde. 2015;2(14):21-31.
19. Tavares CMM, Muniz MP, Elias ADS, Souza MT. Atenção de enfermagem à família do portador de transtorno mental: contribuições para educação permanente. Ciênc Cuid Saúde. 2012;11(4):767-74.

20. Silva HHS, Silva PMC, Azevedo EB, Rocha DC, Costa LFP, Musse JO. Intervenção de enfermagem ao paciente em crise psiquiátrica nos centros de atenção psicossocial. Cogitare Enferm. 2012;17(3):464-70.

21. Lima ICS, Guimarães AB. Perfil das emergências psiquiátricas atendidas em serviços de urgência e emergência hospitalar. $\mathrm{R}$ Interd. 2015;8(2):181-90.

22. Santos ACT, Nascimento YCML, Lucena TS, Rodrigues PMS, Brêda MZ, Santos GF. Mobile service attendance of urgency to psychiatric urgencies and emergencies. J Nurs UFPE online. 2014;8(6):1586-96.

23. Borges CA, Vasconcelos CR, Oselame GB, Dutra DA. O novo perfil profissional do enfermeiro frente ao centro de atenção psicossocial. Rev Med Saúde Brasília. 2016;5(2):217-233.

24. Ferreira WFS, Oliveira EC, Vasconcelos CR, Dutra DA. Direitos humanos da pessoa idosa portadora de esquizofrenia: uma contribuição da enfermagem. Rev Saúde Desenvol. 2017;11(6):219-229.

25. Barbosa MC, Vasconcelos CR, Oselame GB. A percepção do acadêmico de enfermagem sobre a loucura. Rev Enferm Atenção Saúde. 2016;5(2):3-17.

\section{Como citar este artigo:}

Oliveira S, Ferreira WFS, Vasconcelos CR, Dutra DA. O enfrentamento da equipe de enfermagem em atendimentos a pacientes em crise psicótica. Rev. Aten. Saúde. 2017;15(53):50-56. 